Orthopäde 2019 · 48:563-571

https://doi.org/10.1007/s00132-019-03753-2

Online publiziert: 23. Mai 2019

(c) Der/die Autor(en) 2019

Bei der Behandlung eines Sarkoms im Kindesalter sind Wachstumsprothesen eine wichtige Alternative zu Amputation und Umkehrplastik. Mittlerweile gibt es mehrere Systeme, die entweder invasiv oder nichtinvasiv verlängerbar sind. Das Ziel dieses Beitrages ist es, neben der Indikationsstellung und den Erfolgsaussichten die altersspezifischen Probleme aufzuzeigen und Lösungsmöglichkeiten darzustellen.

\section{Einleitung}

Während Amputationen noch vor 40 Jahren die zentrale Säule in der chirurgischen Sarkombehandlung bei Lokalisation in den Extremitäten darstellten, wurde nach Einführung effizienter adjuvanter und neoadjuvanter Chemotherapien die Indikation $\mathrm{zu}$ ablativen Verfahren sukzessive zugunsten der Extremitäten erhaltenden Chirurgie geändert. Für die Wiederherstellung des resezierten Knochensegmentes wurden zahlreiche Rekonstruktionsverfahren angewendet, wobei sich für die Rekonstruktion von Gelenken der endoprothetische segmentale Gelenksersatz durchgesetzt hat. Erfahrungen, die mit individuell angefertigten Endoprothesen gewonnen wurden, konnten in die Entwicklung von modularen Tumorsystemen eingebracht werden, die eine unmittelbare Verfügbarkeit im Operationssaal gewährleisteten.

Bei Kindern und Jugendlichen ist die endoprothetische Rekonstruktion des resezierten Knochenabschnittes respektive Gelenkteiles durch die Wegnahme zumindest einer Wachstumsfuge

R. Windhager · P. Funovics · J. Panotopoulos · G. Hobusch · M. Schinhan

Universitätsklinik für Orthopädie und Unfallchirurgie, Medizinische Universität Wien, Wien, Österreich

\title{
Wachstumsprothesen nach Sarkomresektionen im Kindes- und Jugendalter
}

und der daraus resultierenden Beinlängendifferenz weiter erschwert. Mit der Einführung von teleskopartig verlängerbaren Endoprothesen, den sogenannten Wachstumsprothesen, sollte diesem Umstand Rechnung getragen werden und die Indikation zur Extremitätenerhaltung auch in das Kindesalter ausgedehnt werden. Interessant ist, dass bereits 1976 erste Wachstumsprothesen entwickelt wurden, nur 1 Jahr nach erstmaliger Anwendung der Umkehrplastik nach Borgreeve zur Behandlung kniegelenksnaher Sarkome im Wachstumsalter an der Universitätsklinik für Orthopädie in Wien [15]. Die deutlich erhöhte Reoperationsrate von Wachstumsprothesen sowie die eingeschränkte Sportfähigkeit lassen die Umkehrplastik auch heutzutage als wichtige Alternative zur Sarkombehandlung im Kleinkindesalter erscheinen.

\section{Indikationsstellung}

Wachstumsprothesen kommen nahezu ausschließlich im Bereich der unteren Extremität und hier vor allem bei kniegelenksnahen Tumoren infrage. Verkürzungen nach endoprothetischem Ersatz im Bereich der oberen Extremität haben nur geringe bis gar keine funktionellen Nachteile und werden auch vom kosmetischen Aspekt der Verkürzung sehr gut toleriert.

Unter Berücksichtigung onkologischchirurgischer Kriterien sollte eine ausreichende Muskelerhaltung gewährleistet sein, um ein zufriedenstellendes funktionelles Ergebnis zu sichern und auch die Weichteildeckung der Prothese zu gewährleisten. Durch Erstellung einer
Wachstumsprognose und Kalkulation des fehlenden Wachstums aus der resezierten Wachstumsfuge wird der notwendige Verlängerungshub der Prothese errechnet, wobei dieser besonders bei kleinen Tumoren und damit kurzen Resektionsprothesen gering ausfallen kann [1, 5, 19]. Prinzipiell werden Wachstumsprothesen nur dann angewendet, wenn das errechnete Defizit aus der resezierten Fuge mehr als $4 \mathrm{~cm}$ beträgt. Manche Zentren sehen die Verwendung einer Resektionsprothese auch bei $3 \mathrm{~cm}$ als gerechtfertigt an. In allen anderen Fällen wird durch Verwendung einer längeren Resektionsprothese ein Beinlängenüberschuss von etwa 1 bis max. $2 \mathrm{~cm}$ nach erfolgter Tumorresektion erzielt, der durch Schuhausgleich auf der kontralateralen Seite ausgeglichen wird.

Dieser Schuhausgleich wird anschließend auf der operierten Seite getragen, sobald das gesunde kontralaterale Bein durch aufholendes Wachstum wieder eine Überlänge erreicht hat, die aber auch durch eine Epiphyseodese auf dieser Seite dauerhaft verhindert werden kann. Da der Verlängerungshub von der Länge der Prothese und damit des

\begin{tabular}{|ll}
\hline Abkürzungen \\
\hline GMRS & $\begin{array}{l}\text { Global Modular Replacement } \\
\text { System }\end{array}$ \\
\hline HRMS & $\begin{array}{l}\text { Howmedica Modular Reconstruc- } \\
\text { tion System }\end{array}$ \\
\hline KMFTR & $\begin{array}{l}\text { Kotz Modular Femur Tibia } \\
\text { Reconstruction System }\end{array}$ \\
\hline MRT & Magnetresonanztomographie \\
\hline MSTS & Musculoskeletal Tumor Society \\
\hline
\end{tabular}



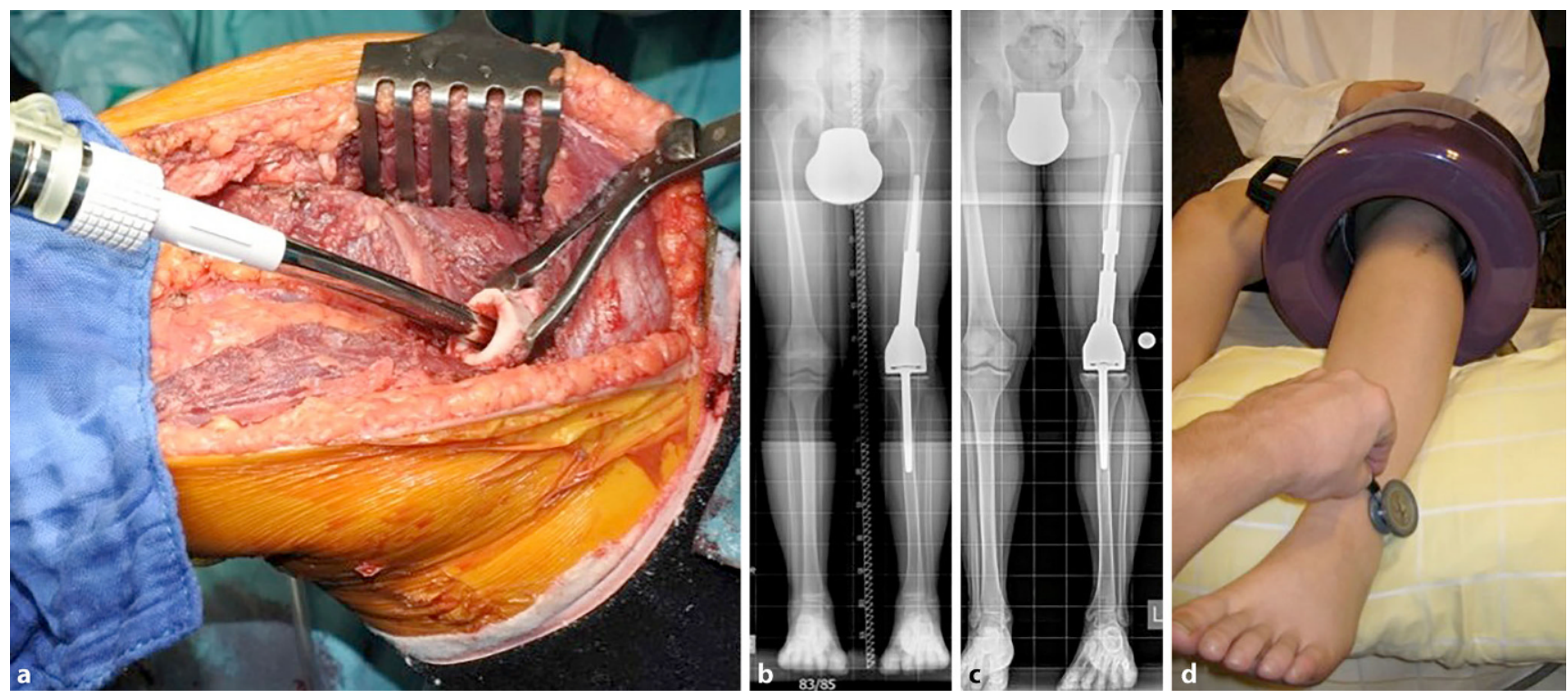

Abb. 1 ॥ Patient, der mit einer nichtinvasiv verlängerbaren Prothese im Alter von 13 Jahren nach Resektion eines Osteosarkoms im distalen Femur versorgt wurde. a Zementfreie Implantation der femoralen Verankerung. Die mit Hydroxylapatit beschichtete osteointegrative Fläche ist kurzstreckig angelegt, um ein „stress shielding“ zu verhindern. b Ausgangssituation im Alter von 13 Jahren. Der tibiale Verankerungsstiel ist glatt, um ein Restwachstum aus der proximalen Tibiafuge zu ermöglichen. c Am Ende des Wachstums wurde eine Verlängerung von insgesamt $53 \mathrm{~mm}$ (32 mm Verlängerung aus der Prothese und $21 \mathrm{~mm}$ Restwachstum aus der proximalen Tibia) erreicht. $\mathrm{d}$ Die Verlängerung der Prothese wird ohne Narkose mit einer Magnetfeldspule bewerkstelligt und die Aktivierung der Prothesenverlängerung durch Abhören der Geräusche, die hierbei entstehen, überprüft

resezierten Knochens abhängt, ist allein dadurch eine Alterslimitierung auf etwa 7-8 Jahren gegeben. So beträgt der Hub bei einer häufig verwendeten nichtinvasiven Wachstumsprothese und einer Länge von $170 \mathrm{~mm}$, lediglich $50 \mathrm{~mm}$ und steigt auf $70 \mathrm{~mm}$ bei $190 \mathrm{~mm}$ Resektion und $90 \mathrm{~mm}$ bei $210 \mathrm{~mm}$ Resektionslänge an. Bei invasiv verlängerbaren modularen Systemen besteht die Möglichkeit der operativen Verkürzung des Wachstumsmoduls und Implantation eines zusätzlichen Segmentersatzes, um die Verlängerung weiter fortzusetzen. Bei aufwändigen nichtinvasiv verlängerbaren Prothesen bedürfte es eines kompletten Prothesenwechsels, da die Prothese meist mit dem Verankerungsstiel im Knochen direkt verbunden ist. Bei sehr kleinen Kindern bedeutet dies, dass während der Wachstumsphase ein oder mehrere Prothesenwechsel erforderlich werden, um entstehende Wachstumsdefekte und Distanzen von mehr als $10 \mathrm{~cm}$ auszugleichen, sodass die Indikation hier kritisch zu stellen ist.

\section{Verlängerungsmechanismus}

Frühe Modelle (1982-1988) der Firma Stanmore (Hertford, United Kingdom) wurden durch Einbringen von Metallkugeln in einen zentralen teleskopartig ausziehbaren Zylinder oder durch Interposition von geschlitzten Zylindern um einen zentralen Stab (1988-1994) verlängert.Spätere Modelle der Firma Stanmore oder die Lewis-Wachstumsprothese bestanden aus einer zentralen Spindel, die invasiv über ein Kegelrad mit einem Schraubendreher verlängert wurde [24]. Dieses System galt als verlässlich, bedurfte jedoch mehrerer Verlängerungsschritte zwischen 10 und $15 \mathrm{~mm}$, um die Prothese zu extendieren und die Beinlänge auszugleichen. Ein System dieser Art bietet weiterhin die Firma Stryker Howmedica (Kalamazoo, MI, USA) im Rahmen des GMRS-Systems als auswechselbares Modul an [23]. Der Vorteil eines solchen Modulsystems liegt in der Möglichkeit, das Verlängerungsmodul nach Wachstumsabschluss und Ausgleich der Beinlänge eventuell gegen ein Standardmodul auszutauschen, wenngleich von allen Firmen Wachs- tumsprothesen als definitive Implantate deklariert sind. Dies setzt die Verwendung eines osteointegrativen Verankerungsstieles im korrespondierenden Gelenkpartner trotz intakter Wachstumsfuge voraus. Wird hingegen ein glatter Verankerungsstiel mit Perforation der gegenüberliegenden Wachstumsfuge verwendet, ist ein weiteres Wachstum aus dieser Fuge möglich, jedoch ist ein Wechsel auf ein Implantat mit osteointegrativer Oberfläche nach Wachstumsende einzuplanen. Das Restwachstum, das sich aus diesen sogenannten passiven Wachstumsteilen ergibt, beträgt im Schnitt 69\% (43-100\%) im Vergleich zu einer nichtperforierten Wachstumsfuge, wobei die große Variabilität des Restwachstums nicht erklärbar und damit voraussagbar ist $[1,4,5]$. Hierdurch kann es zu einer Verschiebung der Gelenklinie kommen, da die Beinlängendifferenz mit der verlängerbaren Prothese ausgeglichen wird.

Eine weiterentwickelte Variante dieses Systems sollte eine nichtinvasive Verlängerung ohne Energiezufuhr von extern gewährleisten, in dem bei Kniebeugung ein Schalter von einem Stößel 
im Tibiaplateau aus aktiviert wurde, der zur Aktivierung des Verlängerungsmechanismus und Extension der Prothese um $0,056 \mathrm{~mm}$ pro Einheit führte. Die Kontrolle der Verlängerung war bei diesem Mechanismus durch das Wechselspiel zwischen Weichteilspannung und Beugefähigkeit einer Autoregulation unterworfen, die eine überschießende Verlängerung verhinderte [16, 29]. Das System wurde durch verfeinernde Entwicklungsschritte und der damit verbundenen Komplexität nicht nur teurer, sondern auch anfälliger für mechanisches Versagen, sodass die Produktion eingestellt wurde.

Das System Repiphysis (Wright Medical Technology, IMC, Arlington, TN, USA) beinhaltete eine Feder mit Vorspannung, die im Prothesenkörper in einem Kunststoffharz eingebettet war und durch Erwärmen der Prothese in einer MRT-Spule aktiviert wurde. Hierdurch kann sich die Feder langsam ausdehnen und führt zur Verlängerung der Prothese. Das früher in den USA häufig verwendete Prothesensystem musste jedoch wegen hoher Komplikationsraten vom Markt genommen werden [25].

Derzeit stehen vor allem nichtinvasive verlängerbare Prothesen, die einerseits über eine Magnetfeldspule (Stanmore, - Abb. 1a-d) oder aber einen elektrischen Motor, der mittels Induktionsspule über einen subkutan implantierten Sensor betätigt wird (Mutars, Implantcast, Buxtehude, Deutschland), in Verwendung.

\section{Probleme an speziellen Lokalisationen}

Wenngleich - entsprechend der Primärlokalisation der Sarkome - distale Femurresektionen die häufigste Indikation für eine Wachstumsprothese darstellen und auch hier die besten funktionellen Ergebnisse zeigen, so wird vereinzelt eine Anwendung im Bereich des proximalen Femurs bis hin zum totalen Ersatz des Femurs respektive der proximalen Tibia notwendig. Am proximalen Femur besteht einerseits die Problematik der Reinsertion der pelvitrochantären Muskulatur, die für das funktionelle Ergebnis entscheidend ist, ebenso wie die

Orthopäde 2019 · 48:563-571 https://doi.org/10.1007/s00132-019-03753-2

(c) Der/die Autor(en) 2019

R. Windhager · P. Funovics · J. Panotopoulos · G. Hobusch · M. Schinhan

\section{Wachstumsprothesen nach Sarkomresektionen im Kindes- und Jugendalter}

Zusammenfassung

Hintergrund. Wachstumsprothesen stellen eine wertvolle Alternative zur Amputation und Umkehrplastik bei Behandlung von Knochen- und Weichteilsarkomen im Kindesalter dar. In den letzten drei Dekaden wurden verschiedene Systeme eingeführt und von invasiv verlängerbaren Prothesen hin zu nichtinvasiv extendierbaren Prothesen weiterentwickelt.

Studienlage. Trotz der langen Zeitspanne finden sich nur 21 Arbeiten in Peer-ReviewJournalen, die relevante Fallzahlen und Ergebnisse präsentieren. In diesen Arbeiten sind 590 Patienten mit einem durchschnittlichen Untersuchungszeitraum von 81,1 Monaten beschrieben, die im Alter von 12,6 Jahren mit einer Wachstumsprothese versorgt worden waren. Bei sehr zufriedenstellenden funktionellen Ergebnissen (78,3 von maximal 100 MSTS-Punkten) war dennoch eine hohe Komplikationsrate mit 27,3\% Infektionen und $22,4 \%$ mechanischen Versagen zu verzeichnen.

Komplikationen. Das über einen langen Nachbeobachtungszeitraum kontinuierlich steigende Infektionsrisiko stellt wohl den größten Nachteil dieser Methodik dar, weswegen diese Information ausführlich bei der Indikationsstellung und Differenzialindikation zu ablativen Verfahren mit den Betroffenen und den Angehörigen erörtert werden muss.

Schlüsselwörter

Osteoasarkom · Ewingsarkom · Beinverlängerung · Extremitätenrekonstruktion . Endoprothese

\section{Growing prostheses after sarcoma resection in children and adolescents}

\section{Abstract}

Background. Growing prostheses are regarded as a valuable alternative to amputation and rotationplasty for the treatment of primary malignant bone and soft-tissue sarcomas in childhood. During the last three decades different devices have been introduced and technically improved from invasively to non-invasively extendable prostheses.

The current situation of studies. Despite the long period, only 21 peer-reviewed publications could be detected containing relevant numbers and results. In these papers, 590 patients with mean follow-up times of 81.1 months were reported who had been fitted with growing prostheses at the age of
12.6 years. Besides satisfactory functional results (78.3 out of 100 MSTS points) there was a high complication rate of $27.3 \%$ infections and $22.4 \%$ mechanical failure. Complications. This increasing risk of infection over a long follow-up period, represents the biggest drawback of this method and, therefore, needs to be discussed extensively with the patients and parents when considering this procedure as an alternative to ablative surgery.

\section{Keywords}

Osteosarcoma · Ewingsarcoma · leg elongation · limb salvage $\cdot$ Endoprosthesis
Rekonstruktion des Femurkopfes, wofür, wenn immer möglich, ein bizentrischer Femurkopfersatz zur Anwendung kommen sollte. Bei nicht exakter Einpassung des Femurkopfes kann es aufgrund geschwächter Abduktoren und der durch Verlängerung bedingten Druckerhöhung im Bereich des Pfannenerkers zu einer Dezentrierung des Kopfes mit sekundärer Dysplasie kommen, die reorientierende Eingriffe am Becken erfor- derlich machen können. An der proximalen Tibia hat sich die Rekonstruktion des Ligamentum patellae mit einem lokal gestielten Gastroknemiuslappen und temporärer Augmentation dieses Konstruktes in Form einer Rahmennaht mit resorbierbaren Materialien bestens bewährt (• Abb. 2a-d).

Überschießende Weichteilreaktionen werden vor allem bei Anwendung im Alter unter 10 Jahren beobachtet und 

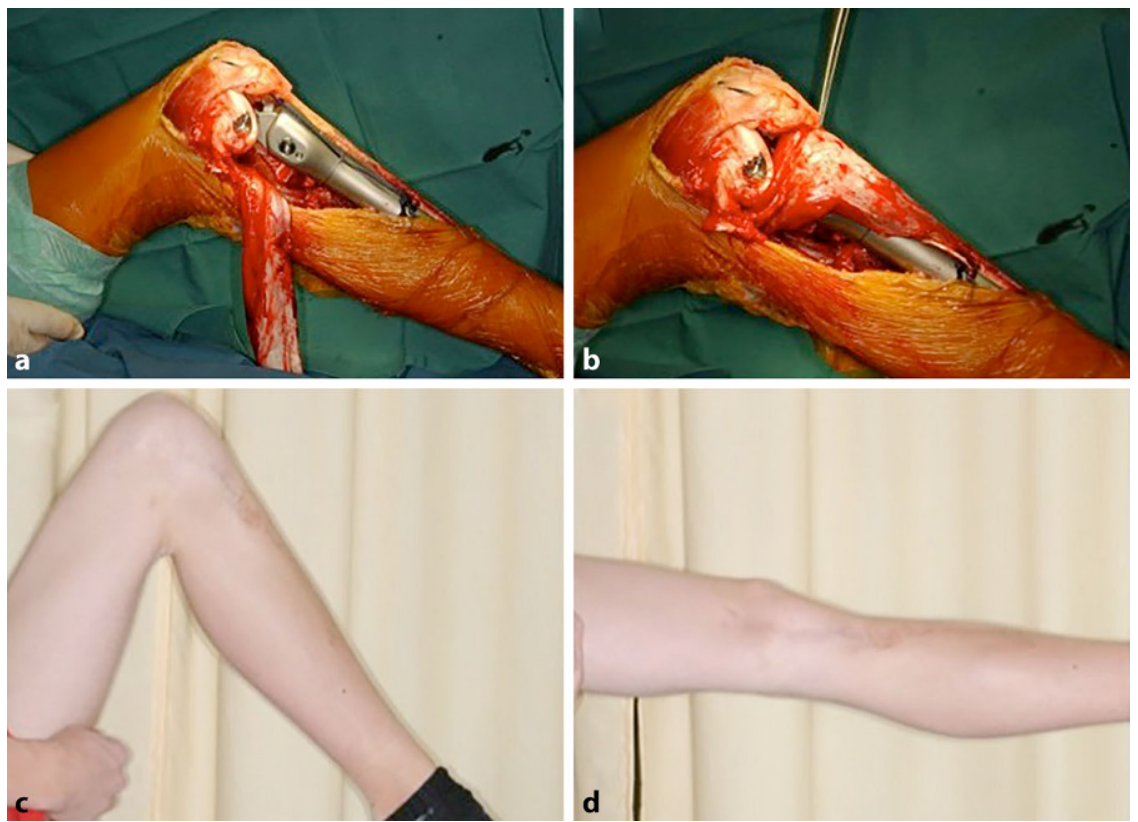

Abb. $2 \Delta$ a Rekonstruktion der proximalen Tibia mit manuell verlängerbarer Wachstumsprothese. Der Musculus gastrocnemius medial ist distal abgelöst und bis zum Gefäßstiel proximal freipräpariert. b Der proximal gefäßgestielte Muskel wird über die Prothese gelegt und die Faszie mit dem Ligamentum patellae, welches mit einer resorbierbaren Rahmennaht an die Prothese fixiert wurde, vernäht. c Aktive Flexion. d Aktive Extension ohne Streckdefizit

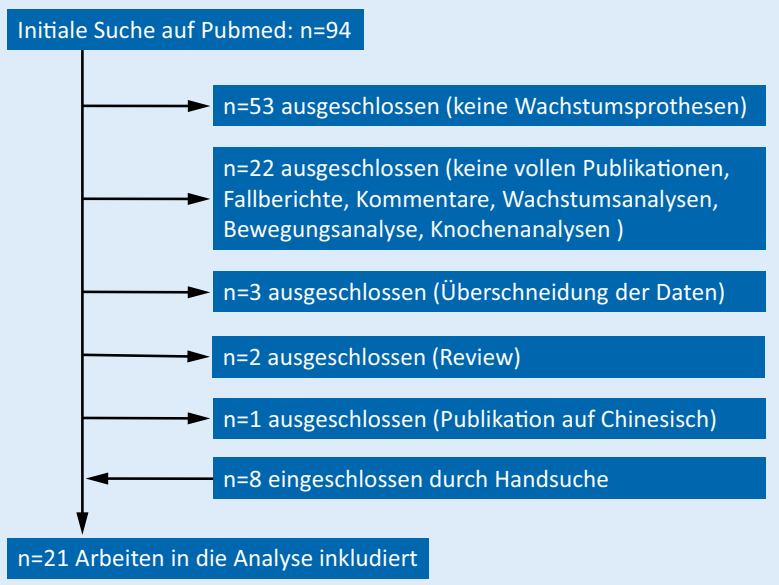

Abb. $3<$ Flow-Chart mit der Übersicht des systematischen Reviews

können bis zur kompletten Gelenkssteife führen [30]. In diesen Fällen ist auch eine Verlängerung der Prothese, die einen zufriedenstellenden Bewegungsumfang am betroffenen Gelenk voraussetzt, nicht mehr möglich, sodass eine Arthrolyse mit Lösung der umgebenden Muskulatur und Resektion des mitunter sehr ausgeprägten Narbengewebes um die Prothese erforderlich ist, um weitere Verlängerungsschritte zu garantieren.

\section{Ergebnisse in der Literatur}

Es wurde ein systematisches Review in Pubmed unter Verwendung folgender Suchkriterien durchgeführt: „growing prostheses“ and „children“ or „KMFTR" or „repiphysis“ or „Mutars“ or „Stanmore“ or "Pafford-Lewis“ and „osteosarcoma“.

Hierbei wurden 94 Publikationen gefunden, von denen 53 vorweg exkludiert wurden, weil Wachstumsprothesen in diesen Publikationen nicht verwendet worden sind. Weitere 22 Veröffentlichungen wurden nicht berücksichtigt, weil es sich nur um Fallberichte oder Kommentare handelte oder aber nur Analysen zu Wachstum, Bewegung oder Knochen enthielten. Da sich die Daten überschnitten haben wurden 3 Publikationen herausgenommen, sowie 2 weitere, da es sich in einem Fall um einen Review handelte und die zweite Arbeit nur auf Chinesisch verfügbar war. Weitere 8 Arbeiten wurden durch Handsuche inkludiert (- Abb. 3). Somit verblieben 21 Arbeiten, die in - Tab. 1 aufgelistet sind [2-4, $6,8-13,17,18,20-22,24-28,31]$.

Insgesamt sind in diesen Arbeiten 590 Patienten beschrieben mit einem mittleren Nachuntersuchungszeitraum von 81,1 Monaten. Der Mittelwert der funktionellen Ergebnisse liegt, gemessen am MSTS-Score [7], bei 78,3 von maximal 100 Punkten.

Das Durchschnittsalter der Patienten lag bei 12,6 Jahren. Die wichtigsten Komplikationsraten betreffend Infektionen respektive mechanisches Versagen betrugen $27,3 \%$ bzw. $22,4 \%$.

\section{Invasiv verlängerbare Wachs- tumsprothesen}

Erste Ergebnisse über Wachstumsprothesen in Peer-Review-Journalen wurden von Jeff Eckardt 1993 publiziert, allerdings nur mit einem durchschnittlichen Nachuntersuchungszeitraum von 3,1 Jahren [6]. Dies ist auch die einzige Publikation, in der die Mehrzahl der Patienten mit einer Pafford-Lewis-Prothese (LEAP Design - Lewis Expandable Adjustable Prosthesis, Arlington, TN, USA) versorgt worden sind. Trotz des kurzen Beobachtungszeitraumes traten in 10 Fällen Komplikationen auf, von denen 7 prothesenassoziiert waren, respektive durch den Verlängerungsmechanismus verursacht wurden. Die von den Autoren geforderte Überarbeitung des Designs führte schließlich auch dazu, dass aufgrund der Weiterentwicklungen dieser Prothesentyp in der Literatur nicht weiter beschrieben ist.

Eine dieser Weiterentwicklungen wurde im Rahmen des HRMS (Howmedica Modular Reconstruction System), welches 1987 eingeführt wurde [14], 
fortgesetzt. Bei dem System wurde die Verlängerung wie bei der LEAP-Prothese minimal-invasiv durch einen Schraubmechanismus umgesetzt und bedurfte mehrerer Verlängerungsschritte zwischen 10 und $15 \mathrm{~mm}$. Erste Ergebnisse wurden 1995 von Schiller et al. publiziert [23] und die Ergebnisse von 6 der insgesamt 20 versorgten Patienten, die das Wachstum bereits abgeschlossen hatten, analysiert. Bei einem durchschnittlichen Operationsalter von 11 Jahren $(9,2-13,7)$ und einem durchschnittlichen Nachuntersuchungszeitraum von 6,3 Jahren (4,3-7,6) wurden 7 Revisionen erforderlich, wobei in 3 Fällen in diesem mittleren Beobachtungszeitraum eine tiefe Infektion auftrat. Für eine durchschnittliche Verlängerungsstrecke von $13,15 \mathrm{~cm}(4,5-19,5)$ waren insgesamt 53 geplante Eingriffe notwendig.

In einer japanischen Sammelstudie wurden 26 von 28 Patienten, die ebenso mit einer HMRS-Wachstumsprothese versorgt worden waren, analysiert. Bei einer durchschnittlichen Nachuntersuchungszeit von 5 Jahren und 1 Monat (1-15 Jahre) fand sich in diesem Kollektiv lediglich eine Infektion. Die durchschnittliche Gesamtverlängerung betrug allerdings nur $35,4 \mathrm{~mm}$, wofür im Schnitt 2,1 Eingriffe erforderlich waren. Diese geringe Zahl an Revisionseingriffen dürfte auch der Grund für die sehr geringe Infektionsrate gewesen sein [31].

\section{I) Die Autoren verweisen auf die Notwendigkeit der exakten präoperativen Aufklärung}

Die längsten Ergebnisse mit einer HMRSWachstumsprothese finden sich in der Arbeit von Schinhan et al. 2015 [21]. In dieser Arbeit wurden 71 Patienten, die mit einer Wachstumsprothese im Alter von 10,1 Jahren (alle HMRS mit Ausnahme einer Mutars-Prothese und einer LEAP-Prothese) versorgt worden waren, über durchschnittlich 131,6 Monate (27,2-281,8 Monate) nachverfolgt. Interessanterweise fand sich in den meisten Fällen ( $46 \%$ der gesamten Komplikationen) ein sogenanntes Weichteilversagen, welches entweder den Streckapparat oder aber eine Instabilität im Bereich der Hüfte mit sekundärer Dysplasie und Dislokation betraf. In $16 \%$ der Fälle berichten die Autoren über die Notwendigkeit einer Resektion einer derben Narbe um die gesamte Prothese, die zu einer massiven Bewegungseinschränkung und damit einer Unmöglichkeit der weiteren Verlängerung geführt hat. Die weitere Analyse zeigte ein strukturelles Versagen in $28 \%$ der Fälle sowie eine Infektionsrate von $17 \%$ und aseptische Lockerungen in nur $8 \%$ aller Fälle. Letzteres ist wohl das überraschendste Ergebnis, wird doch von der Annahme ausgegangen, dass durch das diametrale Wachstum des Knochens die aseptische Lockerung bei Kindern deutlich häufiger wäre. Die Studie zeigt jedoch auch erstmals, dass für die Erzielung einer durchschnittlichen Verlängerung von $70,8 \mathrm{~mm}, 4,4$ Verlängerungseingriffe pro Patient erforderlich waren, ebenso wie 2,5 Operationen pro Patient zur Behandlung der insgesamt 184 Komplikationen bei 58 Patienten. Aufgrund dessen verweisen die Autoren auch explizit auf die Notwendigkeit der exakten präoperativen Aufklärung über diese Folgeeingriffe und auch darauf, dass weitere Verbesserungen in den einzelnen Bereichen nur im direkten Vergleich mit den vorliegenden Langzeitergebnissen möglich sind. Dennoch ist aufgrund der Verlässlichkeit dieses Verlängerungsmechanismus das System weiterhin im Einsatz.

\section{Nichtinvasiv verlängerbare Wachstumsprothesen}

Die Diskrepanz zwischen Kurzzeitergebnissen und mittelfristigen Ergebnissen wird auch bei der Analyse des Systems Repiphysis evident. Das System, das früher unter dem Namen Phenix (Phenix Medical, Paris, Frankreich) verwendet wurde, basiert auf einer Speicherung der Expansionsenergie in Form einer Feder, die durch nichtinvasive Erwärmungen eines Polyethylenschlauches freigegeben und damit expandiert wird. Erste Ergebnisse bei einem Nachuntersuchungszeitraum von 14 Monaten wurden 2001 von Wilkins et al. bei 6 Patienten ohne prothesenbezogene Komplikationen berichtet [28]. Weitere 18 Prothesen wurden bei 15 pädiatrischen Patienten (drei Revisionen) im Jahr 2003 von Neel et al. analysiert und auch durchaus optimistische Ergebnisse wiedergegeben [18]. Spätere Ergebnisse zeigen jedoch mit Zunahme der Frequenz und des Nachuntersuchungszeitraumes eine deutliche Zunahme der Komplikationen.

Im Jahr 2010 berichteten Saghieh et al. von 17 Patienten, die mit einer Repiphysis für durchschnittlich 61,7 Monate beobachtet wurden und insgesamt 38 Verlängerungssitzungen mit durchschnittlich $8,6 \mathrm{~mm}$ erfuhren [20]. 12 dieser $17 \mathrm{~Pa}$ tienten erlitten Komplikationen, wobei 6 mechanischer Natur waren und in 3 Fällen Infektionen auftraten. In 7 Fällen wurde eine Revision erforderlich und in 3 Fällen eine neue Prothese eingesetzt.

\section{) Selbst nichtinvasive Verlängerungsmechanismen von Wachstumsprothesen haben eine hohe Komplikationsrate}

Ruggieri et al. verglichen 2013 bei 32 Patienten die Versorgungen mit 10 HMRS-, 15 Repiphysis- und 7 Stanmore-Prothesen und zeigten eine signifikant höhere Standzeit der HMRS- in Vergleich zur Repiphysis-Prothese [22]. Die Autoren schlossen, dass selbst nichtinvasive Verlängerungsmechanismen von Wachstumsprothesen mit einer durchwegs hohen Komplikationsrate behaftet sind.

Cipriano et al. beschrieben 2015 bei 6 von 12 Patienten, die mit einer Repiphysis-Prothese im Alter von 10,1 Jahren versorgt und durchschnittlich 72 Monate (26-119) nachbeobachtet wurden, 6 aseptische Lockerungen mit massivem Knochenverlust im Bereich des Verankerungsstieles. Die wohl schlechtesten Ergebnisse wurden schließlich in der Arbeit von Staals et al. 2015 veröffentlicht [25]. Von den 15 Patienten, die sie mit einer Repiphysis-Prothese versorgt hatten, konnten 10 mehr als 5 Jahre (durchschnittlich 104 Monate) nachuntersucht werden. Erstmals wurden auch hier Schmerzen während der nichtinvasiven Verlängerung beschrieben, sodass weitere Verlängerungen unter Anästhesie durchgeführt werden mussten. Bei den Patienten wurden insgesamt 9 Revisionsoperationen durchgeführt, von denen 8 durch 


\section{Leitthema}

Tab. 1 Berücksichtigte Arbeiten aus den Peer-Review-Publikationen

\begin{tabular}{|c|c|c|c|c|c|c|c|c|c|c|c|}
\hline Autor & Jahr & Referenz & Prothesentyp & $\begin{array}{l}\text { Fall- } \\
\text { zahl }\end{array}$ & $\begin{array}{l}\text { Nachunter- } \\
\text { suchungszeit- } \\
\text { raum } \\
\text { (Monate) }\end{array}$ & $\begin{array}{l}\text { Infektionen } \\
\text { (Anzahl der } \\
\text { Patienten) }\end{array}$ & $\begin{array}{l}\text { Mechanisches } \\
\text { Versagen (An- } \\
\text { zahl der Patien- } \\
\text { ten) }\end{array}$ & $\begin{array}{l}\text { Ver- } \\
\text { stor- } \\
\text { ben }\end{array}$ & $\begin{array}{l}\text { Lost of } \\
\text { follow } \\
\text { up }\end{array}$ & $\begin{array}{l}\text { MSTS } \\
\text { Score }\end{array}$ & $\begin{array}{l}\text { Alter } \\
\text { bei } \\
\text { Opera- } \\
\text { tion }\end{array}$ \\
\hline Gilg et al. & 2018 & [8] & Stanmore & 21 & 70 & 10 & 4 & 13 & 0 & 76 & 20 \\
\hline $\begin{array}{l}\text { Medellin } \\
\text { et al. }\end{array}$ & 2018 & [17] & Stanmore & 24 & 158 & 2 & 5 & 0 & 0 & 78 & 11 \\
\hline $\begin{array}{l}\text { Tsagozis } \\
\text { et al. }\end{array}$ & 2018 & [27] & Stanmore & 42 & 72 & 12 & 12 & 2 & 0 & 57 & 10 \\
\hline Bus et al. & 2017 & [2] & Mutars & 105 & $\begin{array}{l}\text { Von } \\
\text { 1995-2010, } \\
\text { min. } 5 \text { Jahre }\end{array}$ & 15 & 15 & 37 & 4 & $x$ & 36 \\
\hline Gilg et al. & 2016 & [9] & Stanmore & 50 & 64 & 10 & 4 & 5 & 0 & 80 & 10 \\
\hline $\begin{array}{l}\text { Grimer } \\
\text { et al. }\end{array}$ & 2016 & [11] & Stanmore & $\begin{array}{l}46 \\
\text { aus } \\
564\end{array}$ & 353 & 60 & $x$ & 302 & 32 & $x$ & 21 \\
\hline $\begin{array}{l}\text { Torner } \\
\text { et al }\end{array}$ & 2016 & [26] & Mutars & 7 & 65 & 1 & 0 & 2 & 0 & 89 & 9 \\
\hline $\begin{array}{l}\text { Cipriano } \\
\text { et al. }\end{array}$ & 2015 & [3] & Repiphysis & 12 & 72 & 1 & 3 & 2 & 0 & 67 & 10 \\
\hline Staals et al & 2015 & [25] & Repiphysis & 15 & 104 & 1 & 9 & 5 & 0 & 81 & 8 \\
\hline $\begin{array}{l}\text { Schinhan } \\
\text { et al. }\end{array}$ & 2015 & [21] & $\begin{array}{l}\text { KMFTR: } 69 \\
\text { Mutars: } 1, \text { Paf- } \\
\text { ford-Lewis: } \\
1\end{array}$ & 71 & 132 & 31 & 52 & 12 & 0 & 88 & 10 \\
\hline $\begin{array}{l}\text { Ruggieri } \\
\text { et al. }\end{array}$ & 2013 & [22] & $\begin{array}{l}\text { KMFTR: 10, } \\
\text { Repiphysis: } 15 \text {, } \\
\text { Stanmore: } 7\end{array}$ & 32 & 49 & 3 & 2 & 7 & 0 & 79 & 9 \\
\hline $\begin{array}{l}\text { Henderson } \\
\text { et al. }\end{array}$ & 2012 & [13] & $\begin{array}{l}\text { KMFTR: } 17 \text {, } \\
\text { Stanmore: } 8\end{array}$ & 17 & $\begin{array}{l}\text { Von } \\
2001-2007\end{array}$ & 1 & 1 & 0 & 0 & $x$ & 11 \\
\hline $\begin{array}{l}\text { Saghieh } \\
\text { et al. }\end{array}$ & 2010 & [20] & Repiphysis & 17 & 62 & 3 & 6 & 1 & 0 & 90 & 11 \\
\hline $\begin{array}{l}\text { Yoshida } \\
\text { et al. }\end{array}$ & 2008 & [31] & $\begin{array}{l}\text { KMFTR: } 26 \text {, } \\
\text { Lewis: } 1 \text {, Stan- } \\
\text { more: } 1\end{array}$ & 28 & 61 & 1 & 3 & 3 & 0 & $x$ & 10 \\
\hline $\begin{array}{l}\text { Gupta } \\
\text { et al. }\end{array}$ & 2006 & [12] & Stanmore & 7 & 20 & 0 & 0 & 1 & 0 & 60 & 12 \\
\hline $\begin{array}{l}\text { Gitelis } \\
\text { et al. }\end{array}$ & 2003 & [10] & Repiphysis & 18 & 25 & 1 & 5 & 0 & 0 & $x$ & 11 \\
\hline Neel et al. & 2003 & [18] & Phenix & 18 & 22 & 7 & 0 & 0 & 0 & 90 & 11 \\
\hline $\begin{array}{l}\text { Wilkins } \\
\text { et al. }\end{array}$ & 2001 & [28] & Phenix & 6 & 14 & 0 & 2 & 0 & 0 & 80 & 15 \\
\hline Cool et al. & 1997 & [4] & Stanmore & 24 & 56 & 1 & 1 & 0 & 0 & $x$ & 10 \\
\hline $\begin{array}{l}\text { Schindler } \\
\text { et al. }\end{array}$ & 1997 & [24] & Stanmore & 18 & 104 & 1 & 1 & 4 & 0 & 78 & 11 \\
\hline $\begin{array}{l}\text { Eckardt } \\
\text { et al. }\end{array}$ & 1993 & [6] & $\begin{array}{l}\text { Lewis: } 10 \text {, Tech } \\
\text { Medica: } 2\end{array}$ & 12 & 37 & 0 & 7 & 4 & 0 & 88 & 9 \\
\hline \multirow[t]{2}{*}{ - } & \multirow[t]{2}{*}{-} & \multirow[t]{2}{*}{-} & \multirow[t]{2}{*}{-} & 590 & 81,1 & 7,7 & 6,6 & \multirow[t]{2}{*}{-} & \multirow[t]{2}{*}{-} & 78,3 & 12,6 \\
\hline & & & & $\begin{array}{l}\text { Sum- } \\
\text { me }\end{array}$ & Mittelwert & Mittelwert & Mittelwert & & & $\begin{array}{l}\text { Mittel- } \\
\text { wert }\end{array}$ & $\begin{array}{l}\text { Mittel- } \\
\text { wert }\end{array}$ \\
\hline
\end{tabular}


Hier steht eine Anzeige.

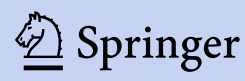


Implantatversagen und eine durch aseptische Lockerung bedingt waren. Auch der Knochenverlust wird hervorgehoben, der bei 5 Fällen zu Knochenaugmentationen während der Revisionsoperation geführt hat. In der Conclusio nehmen die Autoren von der weiteren Verwendung dieser Prothese abstand. Aufgrund der sich häufenden Negativveröffentlichungen wurde die Prothese schließlich vom Markt genommen.

Mehrere Publikationen beschäftigen sich auch mit der nichtinvasiven Wachstumsprothese der Firma Stanmore, die von außen durch ein elektromagnetisches Feld angetrieben und so verlängert wird. Wie bei anderen Systemen wurden erste Publikationen mit kleiner Fallzahl und kurzer Nachbeobachtungszeit veröffentlicht. So berichten Gupta et al. 2006 von 7 Fällen, die für nur 20,2 Monate nachbeobachtet und deren Prothese um lediglich $25 \mathrm{~mm}$ im Durchschnitt verlängert worden ist [12]. Auch nach diesen ermutigenden Kurzzeitergebnissen wurde von Gilg und Mitarbeitern 2016 über die Morbidität der nichtinvasiven Wachstumsprothese bei 50 Kindern mit 51 Prothesen berichtet [9]. Beim Durchschnittsoperationsalter von 10,4 Jahren und einer Nachbeobachtungszeit von 64 Monaten (20-145) war die Überlebensrate der Prothese bei $81,7 \%$ nach 3 Jahren und 61,6\% nach 6 Jahren.

\section{》) Silberbeschichtete Implan- tate brachten keinen Benefit in Bezug auf Infektionskontrolle}

Eine tiefe Infektion musste bei 19,6\% nach durchschnittlich 12,5 Monaten festgestellt werden und in 9,8\% fand sich ein Versagen des Verlängerungsmechanismus sowie ein Implantatbruch in 3,9\% der Fälle. Die Zahl der Reoperationen war mit 53 ähnlich wie in anderen Publikationen, wobei die Amputationsrate mit $14 \%$ (3 wegen Lokalrezidiv und 4 wegen Infektionen) eher höher angesetzt ist. Die Autoren heben hervor, dass bei guten funktionellen Ergebnissen und Kompensation der Beinlänge, die Infektion nach wie vor das größte Risiko darstellt, wobei die proximale Tibia das höchste Risiko birgt. Die Ergebnisse der Versorgung mit einer Wachstumsprothese in dieser Region wird in einer eigenen Arbeit von Tsagozis et al. 2018 bei 42 Patienten retrospektiv analysiert [27]. Als Besonderes hervorzuheben ist, dass in dieser Studie die Versorgung mit nichtinvasiven Verlängerungsprothesen mit einer höheren Infektionsrate als bei konventionellen Verlängerungsprothesen behaftet und mit einer höheren Amputationsrate verbunden war. Trotz guter funktioneller Ergebnisse betrug die Prothesenstandzeit nach 5 Jahren lediglich $55 \%$ und nur $25 \%$ nach 10 Jahren. Hervorzuheben ist auch, dass die Versorgung mit silberbeschichteten Implantaten in dieser Lokalisation keinen Benefit in Bezug auf Infektionskontrolle und damit Prothesenüberleben brachte.

Dieser Nachteil der nichtinvasiv verlängerbaren Prothesen konnte in der Analyse von Medellin et al. von 24 totalen Femurversorgungen in einem Nachuntersuchungszeitraum von 13,2 Jahren (7 Monate bis 29,25 Jahre) nicht nachvollzogen werden [17]. Es fanden sich keine Diskrepanzen in Bezug auf verschiedene Komplikationsarten. Allerdings waren in der Gruppe der minimalinvasiv verlängerbaren totalen Femurprothesen eine genauere Einstellung der Beinlängendifferenz, eine bessere Kniegelenksbeweglichkeit und eine verbesserte Gesamtfunktion zu verzeichnen. Die Prothesenstandzeiten nach 5, 10 sowie 20 Jahren betrugen jeweils $79 \%$ für minimal-invasiv verlängerbare Stanmore-Prothesen und $85 \%$ nach 5 sowie $70 \%$ nach 10 Jahren für nichtinvasiv verlängerbare Wachstumsprothesen der Firma Stanmore.

Schließlich wurde in der Arbeit von Gilg et al. die Anwendung der verlängerbaren Prothesen bei Revisionsoperationen mit massiver Beinlängendiskrepanz bei 21 Patienten und einem durchschnittlichen Alter von 20 Jahren (10-41) analysiert [9]. Bei einer Nachuntersuchungszeit von 70 Monaten (17-128) wurde eine durchschnittliche Verlängerung von $51 \mathrm{~mm}$ (5-140) erzielt und eine Prothesenstandzeit von $75 \%$ nach 2 Jahren und $55 \%$ nach 5 Jahren beschrieben.

Mit der Kostenanalyse beschäftigt sich schließlich die Arbeit von Henderson et al. und vergleicht 17 Patienten, die mit einer minimal-invasiv verlängerbaren Prothese und 8 Patienten, die mit einer nichtinvasiv verlängerbaren Prothese versorgt wurden [13]. Unter Zugrundelegung US-amerikanischer Verhältnisse schließen die Autoren, dass nichtinvasiv verlängerbare Prothesen trotz höherer Initialkosten aufgrund fehlender Verlängerungseingriffe günstiger abschneiden als minimal-invasiv verlängerbare Prothesen.

Abschließend muss festgehalten werden, dass trotz intensiver Recherche keine Publikationen über die verlängerbare Prothese von Mutars mit größeren Fallzahlen gefunden werden konnte.

\section{Fazit für die Praxis}

- Wachstumsprothesen stellen eine bewährte Methode und Alternative zu ablativen Verfahren wie Amputation und Umkehrplastik bei der Behandlung von Sarkomen im Kindes- und Jugendalter dar.

- Das über einen langen Zeitraum zu beobachtende hohe Infektionsrisiko bedarf einer ausführlichen Erörterung im Rahmen des Aufklärungsgespräches.

- Aufgrund der vielfältigen Komplikationsmöglichkeiten sollte zur Reduktion der Reoperationsfrequenz diese Versorgung nur in spezialisierten Zentren durchgeführt werden.

\section{Korrespondenzadresse}

\section{Prof. Dr. R. Windhager}

Universitätsklinik für Orthopädie und Unfallchirurgie, Medizinische Universität Wien Währinger Gürtel 18-20, 1090 Wien, Österreich reinhard.windhager@meduniwien.ac.at

Funding. Open access funding provided by Medical University of Vienna.

\section{Einhaltung ethischer Richtlinien}

Interessenkonflikt. R. Windhager ist als Referent bzw. Berater für die Firma Stryker tätig, zu der mittlerweile auch die Stanmore Implants Worldwide gehört, und erhält leistungsbezogene Honorare für Vortragsund Beratungstätigkeit. P. Funovics, J. PanotopouIos, G. Hobusch und M. Schinhan geben an, dass kein Interessenkonflikt besteht. 
Für diesen Beitrag wurden von den Autoren keine Studien an Menschen oder Tieren durchgeführt. Für die aufgeführten Studien gelten die jeweils dort angegebenen ethischen Richtlinien.

Open Access. Dieser Artikel wird unter der Creative Commons Namensnennung 4.0 International Lizenz (http://creativecommons.org/licenses/by/4.0/deed. de) veröffentlicht, welche die Nutzung, Vervielfältigung, Bearbeitung, Verbreitung und Wiedergabe in jeglichem Medium und Format erlaubt, sofern Sie den/die ursprünglichen Autor(en) und die Quelle ordnungsgemäßnennen, einen Link zur Creative Commons Lizenz beifügen und angeben, ob Änderungen vorgenommen wurden.

\section{Literatur}

1. Anderson M, Green WT, Messner MB (1963) Growth and predictions of growth in the lower extremities. JBone Joint Surg Am 45-A:1-14

2. Bus MP, van de Sande MA, Fiocco M, Schaap GR, Bramer JA, Dijkstra PD (2017) What are the longterm results of MUTARS ${ }^{\circledR}$ modular Endoprostheses for reconstruction of tumor resection of the distal femur and proximal tibia? Clin Orthop Relat Res 475(3):708-718

3. Cipriano CA, Gruzinova IS, Frank RM, Gitelis S, Virkus WW (2015) Frequent complications and severe bone loss associated with the repiphysis expandable distal femoral prosthesis. Clin Orthop RelatRes 473(3):831-838

4. Cool WP, Carter SR, Grimer RJ, Tillman RM, Walker PS (1997) Growth after extendible endoprosthetic replacement of the distal femur. J Bone Joint Surg Br 79(6):938-942

5. Dominkus $M$, Krepler P, Schwameis E, Windhager R, Kotz R (2001) Growth prediction in extendable tumor prostheses in children. Clin Orthop Relat Res 390:212-220

6. Eckardt JJ, Safran MR, Eilber FR, Rosen G, Kabo JM (1993) Expandable endoprosthetic reconstruction of the skeletally immature after malignant bone tumor resection. Clin Orthop Relat Res 297:188-202

7. Enneking WF, Dunham W, Gebhardt MC, Malawar M, Pritchard DJ (1993) A system for the functional evaluation of reconstructive procedures after surgical treatment of tumors of the musculoskeletal system. Clin Orthop Relat Res 286:241-246

8. Gilg MM, Gaston $C L$, Jeys L, Abudu A, Tillman RM, Stevenson JD, Grimer RJ, Parry MC (2018) The use of a non-invasive extendable prosthesis at the time of revision arthroplasty. Bone Joint J 100B(3):370-377

9. Gilg $M M$, Gaston $C L$, Parry MC, Jeys L, Abudu $A$, Tillman RM, Carter SR, Grimer RJ (2016) What is the morbidity of a non-invasive growing prosthesis? Bone Joint J 98-B(12):1697-1703

10. Gitelis S, Neel MD, Wilkins RM, Rao BN, Kelly CM, Yao TK (2003) The use of a closed expandable prosthesis for pediatric sarcomas. Chir Organi Mov 88(4):327-333

11. Grimer RJ, Aydin BK, Wafa H, Carter SR, Jeys L, Abudu A, Parry M (2016) Very long-term outcomes after endoprosthetic replacement for malignant tumours of bone. Bone Joint J 98-B(6):857-864

12. Gupta A, Meswania J, Pollock R, Cannon SR, Briggs TW, Taylor S, Blunn G (2006) Non-invasive distal femoral expandable endoprosthesis for limbsalvage surgery in paediatric tumours. J Bone Joint Surg Br 88(5):649-654
13. Henderson ER, Pepper AM, Letson GD (2012) What are estimated reimbursements for lower extremity prostheses capable of surgical and nonsurgical lengthening? Clin Orthop Relat Res 470(4):1194-1203

14. Kotz R, Ritschl P, Kropej D, Capanna R (1990) Cementless modular prostheses. Basic concepts and evolution. Chir Organi Mov 75(1 Suppl):177-178

15. Kotz R, Salzer M (1982) Rotation-plasty for childhood osteosarcoma of the distal part of the femur JBone Joint Surg Am 64(7):959-969

16. Kotz Rl, Windhager R, Dominkus M, Robioneck B, Müller-Daniels H (2000) A self-extending paediatric leg implant. Nature 406(6792):143-144

17. Medellin MR, Fujiwara T, Clark R, Jeys LM (2018) Comparison of minimally invasive and noninvasive systems in lengthening total femoral prostheses. Bone Joint J 100-B(12):1640-1646

18. Neel MD, Wilkins RM, Rao BN, Kelly CM (2003) Early Multicenter experience with a noninvasive expandable prosthesis. Clin Orthop Relat Res 415:72-81

19. Paley D, Bhave A, Herzenberg JE, Bowen JR (2000) Multiplier method for predicting limblength discrepancy. J Bone Joint Surg Am 82A(10):1432-1446

20. Saghieh S, Abboud MR, Muwakkit SA, Saab R, Rao B, Haidar R (2010) Seven-year experience of using Repiphysis expandable prosthesis in children with bone tumors. Pediatr Blood Cancer 55(3):457-463

21. Schinhan $M$, Tiefenboeck T, Funovics $P$, Sevelda $F$, Kotz R, Windhager R (2015) Extendible prosthese for children after resection of primary malignant Bone tumor: twenty-seven years of experience. JBone Joint Surg Am 97(19):1585-1591

22. Ruggieri $P$, Mavrogenis AF, Pala $E$, Romantini M, Manfrini M, Mercuri M (2013) Outcome of expandable prostheses in children. J Pediatr Orthop 33(3):244-253

23. Schiller C, Windhager R, Fellinger EJ, SalzerKuntschik M, Kaider A, Kotz R (1995) Extendable tumour endoprostheses for the leg in children.JBone Joint Surg Br 77(4):608-614

24. Schindler OS, Cannon SR, Briggs TW, Blunn GW (1997) Stanmore custom-made extendible distal femoral replacements. Clinical experience in children with primary malignant bone tumours. J Bone Joint Surg Br 79(6):927-937

25. Staals EL, Colangeli M, Ali N, Casanova JM, Donat DM, Manfrini M (2015) Are complications associated with the Repiphysis $\left({ }^{\circledR}\right)$ expandable distal femoral prosthesis acceptable for its continued use? Clin Orthop Relat Res 473(9):3003-3013

26. Torner F, Segur JM, Ullot R, Soldado F, Domenech P, DeSena L, Knorr J (2016) Non-invasive expandable prosthesis in musculoskeletal oncology paediatric patients for the distal and proximal femur. First results. Int Orthop 40(8):1683-1688

27. Tsagozis P, Parry M, Grimer R (2018) High complication rate after extendible endoprosthetic replacement of the proximal tibia: a retrospective study of 42 consecutive children. Acta Orthop 89(6):678-682. https://doi.org/10.1080/ 17453674.2018.1534320

28. Wilkins RM, Soubeiran A (2001) The phenix expandable prosthesis, early American experience. Clin Orthop Relat Res 382:51-58

29. Windhager R, Bien M, Müller H, Kotz R (1995) Wachstumsprothese für Kinder mit automatischer Verlängerung. Med-orthop Tech 115:152-154

30. Windhager R, Nemethova M, Mutsaers S, Lang S, Kotz R, Kitzmueller E, Lubec G (1998) Evidence for the involvement of the hydroxyl radical in the pathogenesis of excessive connective tissue proliferation in patients with tumor-endoprostheses. Life Sci 62(14):1261-1269

31. Yoshida Y, Iwata S, Ueda T, Kawai A, Isu K, Ryu J (2008) Current state of extendable prostheses for the lower limb in Japan. Surg Oncol 17(2):65-71 